\title{
Effects of a Hole on Uplifting Forces on a Submerged Horizontal Thin Plate
}

Deng How Tsaur

Associate Professor, Department of Harbor and River Engineering, National Taiwan Ocean Universiry, Keelung, Taiwan, R.O.C.

Shyh Rong Her

Graduate Student, Department of Harbor and River Engineering, National Taiwan Ocean University, Keelung, Taiwan, R.O.C.

Follow this and additional works at: https://jmstt.ntou.edu.tw/journal

Part of the Civil and Environmental Engineering Commons

\section{Recommended Citation}

Tsaur, Deng How and Her, Shyh Rong (2021) "Effects of a Hole on Uplifting Forces on a Submerged Horizontal Thin Plate," Journal of Marine Science and Technology. Vol. 4: Iss. 1, Article 9.

DOI: $10.51400 / 2709-6998.2498$

Available at: https://jmstt.ntou.edu.tw/journal/vol4/iss1/9

This Research Article is brought to you for free and open access by Journal of Marine Science and Technology. It has been accepted for inclusion in Journal of Marine Science and Technology by an authorized editor of Journal of Marine Science and Technology. 


\title{
EFFECTS OF A HOLE ON UPLIFTING FORCES ON A SUBMERGED HORIZONTAL THIN PLATE
}

\author{
Deng How Tsaur* and Shyh Rong Her**
}

Keywords: uplifiting force, plate with a hole, hypersingular integral equation, boundary element method.

\section{ABSTRACT}

The effect of a hole on uplifting forces acting on a submerged horizontal thin plate are examined using the boundary element method. To deal with the submerged thin plate case, which is a degenerate boundary problem, a hypersingular integral equation is employed to overcome some troubles when the boundary element method is used. Two-dimensional problems are considered, and the linear gravity wave theory is adopted. Several examples are calculated and the numerical results are presented to show the influence of a hole on uplifting forces.

\section{INTRODUCTION}

Some researchers $[1,2,3]$ have studied the effects of a hole on the uplifting forces exerted on a submerged horizontal plate using the boundary element method. In these researches the plate with a thickness has been mainly solved, however, the results for a thin plate case have not been presented. In a previous paper[4], a method for solving the problem of the uplifting force on a submerged horizontal thin plate has been developed successfully. To solve such problems, the hypersingular integral equation in conjunction with the boundary element technique was employed, because the hypersingular integral equation can be used effectively to solve the degenerate boundary-value problems, such as the crack problems[5] and the cutoff wall in seepage problems[6].

In the present paper, the above work is extended to study the case of a submerged thin plate with a

Paper Received May, 1995. Revised June, 1996. Accepted June, 1996. Author for Correspondence: Deng How Tsaur.

*Associate Professor, Department of Harbor and River Engineering, National Taiwan Ocean University, Keelung, Taiwan, R.O.C.

**Graduate Student, Department of Harbor and River Engineering,

National Taiwan Ocean University, Keelung, Taiwan, R.O.C. hole and to investigate the effect of the hole on the uplifting forces, which are due to the gravity wave acting on the submerged horizontal thin plate. For simplicity, two-dimensional problems and the linear regular wave are considered. Some examples are evaluated, and their results for different values of the ratio of a hole to a plate are shown and compared to illustrate how the hole affects the uplifting forces on a submerged horizontal thin plate.

\section{THEORETICAL FORMULATION}

The definition sketch for this problem is shown in Fig. 1. The cartesian coordinate system is used. The $\mathrm{x}$-axis is along the still water surface, and the $\mathrm{z}$ axis is upward along the vertical wall. The entire domain is divided into two regions by a pseudo boundary.

For the inviscid, incompressible fluid and the irrotational flow, the velocity potential $\Phi(x, z, t)$ satisfies the Laplace equation. When the flow is assumed to be a small amplitude wave motion with an angular frequency $\sigma$, the velocity potential $\Phi(x, z, t)$ can be expressed in a complex form as follows:

$$
\Phi(x, z, t)=\frac{g \zeta}{\sigma} \phi(x, z) \exp (i \sigma t),
$$

where $g$ is the gravitational acceleration, $\zeta$ is the

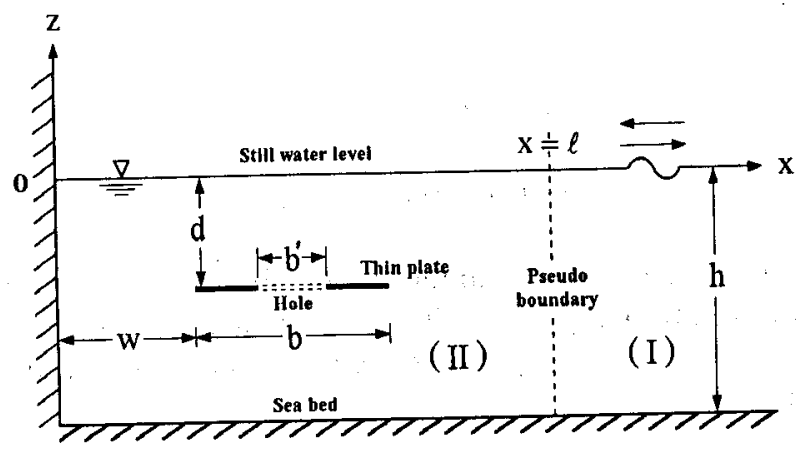

Fig. 1. Definition sketch. 
incident wave amplitude, $t$ is the time, and $\phi(x, z)$ is the potential function.

The potential function $\phi_{I I}(x, z)$ in the region (II) can be determined by the boundary element method with the following boundary conditions:

$$
\begin{aligned}
& \frac{\partial \varphi_{I I}(x, z)}{\partial n}=0, \quad \text { at } x=0, \\
& \frac{\partial \phi_{I I}(x, z)}{\partial z}=\frac{\sigma^{2}}{g} \phi_{I I}(x, z), \quad \text { at } z=0 \\
& \frac{\partial \phi_{I I}(x, z)}{\partial n}=0, \quad \text { at } z=-h \\
& \frac{\partial \phi_{I I}(x, z)}{\partial n}=0, \quad \text { at } z=-d^{+} \text {and } w<x<w \\
& +\frac{\left(b-b^{\prime}\right)}{2}, w+\frac{\left(b+b^{\prime}\right)}{2}<x<w+b \\
& \frac{\partial \phi_{I I}(x, z)}{\partial n}=0, \quad \text { at } z=-d^{-} \text {and } w<x<w \\
& +\frac{\left(b-b^{\prime}\right)}{2}, w+\frac{\left(b+b^{\prime}\right)}{2}<x<w+b
\end{aligned}
$$

and

$$
\begin{aligned}
& \phi_{I I}(x, z)=\phi_{I}(x, z), \text { at } x=\ell, \\
& \frac{\partial \phi_{I I}(x, z)}{\partial x}=\frac{\partial \phi_{I}(x, z)}{\partial x}, \quad \text { at } x=\ell,
\end{aligned}
$$

where $n$ is the unit outward normal; $h$ is the water depth; $d$ is the depth of the location of the plate; $w$ is the distance between the edge of the plate to the vertical wall; $b$ and $b^{\prime}$ are the widths of the plate and the hole, respectively; and $\ell$ is the distance from the vertical wall to the pseudo boundary.

If the pseudo boundary is far away from the plate, the potential function $\phi_{I}(x, z)$ can be given by $[3,7]$

$$
\begin{aligned}
\phi_{I}(x, z) & =\{\exp [i k(x-\ell)] \\
& \left.+K_{r} \exp [-i k(x-\ell)]\right\} Z(z),
\end{aligned}
$$

where $k$ is the wave number that satisfies the dispersion relationship, $K_{r}$ is the coefficient of reflection, and $Z(z)$ is the depth dependency function and is given by

$$
Z(z)=\{\cosh k(h+z) / \cosh k h\} .
$$

\section{INTEGRAL EQUATIONS AND NUMERICAL PROCEDURES}

The potential function $\phi_{I I}(x, z)$ satisfies the Laplace equation, such that it can be expressed in terms of the value of the potential function and its normal derivative on the boundary incorporating the fundamental solution $(\ln r)$ and its normal derivative $\frac{\partial}{\partial n_{s}}(\ln r)$. The boundary integral representation of the potential function $\phi_{I I}\left(\xi^{\prime}, \eta^{\prime}\right)$ at a boundary point $Q\left(\xi^{\prime}, \eta^{\prime}\right)$ is

$$
\begin{aligned}
\alpha \phi_{I I}\left(\xi, \eta^{\prime}\right) & =\int_{\Gamma} \phi_{I I}(\xi, \eta) \frac{\partial}{\partial n_{s}}(\ln r) d \Gamma(s) \\
& -\int_{\Gamma} \frac{\partial \phi_{I I}(\xi, \eta)}{\partial n_{s}}(\ln r) d \Gamma(s),
\end{aligned}
$$

where $r$ is the distance between points $Q^{\prime}\left(\xi^{\prime}, \eta^{\prime}\right)$ and $Q(\xi, \eta), n_{s}$ is the unit outward normal at the source point. $\int$ denotes the Cauchy principal-value integral. As the boundary is smooth, $\alpha$ is equal to $\pi$.

For the numerical computations, the boundary of the region (II) are divided into $N$ constant elements by discretizing the integral equations with the boundary element technique. Equation (11) can then be written in the matrix form,

$$
\begin{aligned}
& {\left[\begin{array}{lll}
\bar{U}_{\Gamma_{n} \Gamma_{n}} & \bar{U}_{\Gamma_{n} \Gamma_{d}^{+}} \bar{U}_{\Gamma_{n} \Gamma_{d}} \\
\bar{U}_{\Gamma_{d}^{+} \Gamma_{n}} & \bar{U}_{\Gamma_{d}^{+} \Gamma_{d}^{+}} \bar{U}_{\Gamma_{d}^{+} \Gamma_{d}^{-}} \\
\bar{U}_{\Gamma_{d}^{-} \Gamma_{n}} & \bar{U}_{\Gamma_{d}^{-} \Gamma_{d}^{+}} \bar{U}_{\Gamma_{d}^{-} \Gamma_{d}^{-}}
\end{array}\right]\left\{\begin{array}{l}
\left(\phi_{I I}\right)_{\Gamma_{n}} \\
\left(\phi_{I I}\right)_{\Gamma_{d}^{+}} \\
\left(\phi_{I I}\right)_{\Gamma_{d}^{-}}
\end{array}\right\}} \\
& =\left[\begin{array}{ccc}
U_{\Gamma_{n} \Gamma_{n}} & U_{\Gamma_{n} \Gamma_{d}^{+}} & U_{\Gamma_{n} \Gamma_{d}^{-}} \\
U_{\Gamma_{d}^{+} \Gamma_{n}} & U_{\Gamma_{d}^{+} \Gamma_{d}^{+}} & U_{\Gamma_{d}^{-} \Gamma_{d}^{-}} \\
U_{\Gamma_{d}^{-} \Gamma_{n}} & U_{\Gamma_{d}^{-}} \Gamma_{d}^{+} & U_{\Gamma_{d}^{-} \Gamma_{d}^{-}}
\end{array}\right]\left\{\begin{array}{c}
\left(\frac{\partial \phi_{I I}}{\partial n}\right)_{\Gamma_{n}} \\
\left(\frac{\partial \phi_{I I}}{\partial n}\right)_{\Gamma_{n}^{+}} \\
\left(\frac{\partial \phi_{I I}}{\partial n}\right)_{\Gamma_{n}^{-}}
\end{array}\right\},
\end{aligned}
$$

where the subscript $\Gamma_{n}, \Gamma_{d}$ denote the nondegenerate boundary and the degenerate boundary, respectively; and $\Gamma_{d}^{+}$and $\Gamma_{d}^{-}$denote the two sides of the degenerate boundary. The elements, $U_{i j}$ and $\bar{U}_{i j}$, of the matrices of eq.(12) are

$$
\begin{aligned}
& U_{i j}=\int_{\Gamma_{j}}(\ln r) d \Gamma\left(s_{j}\right), \\
& \bar{U}_{i j}=\int_{\Gamma_{j}} \frac{\partial}{\partial n_{s}}(\ln r) d \Gamma\left(s_{j}\right)-\pi \delta_{i j},
\end{aligned}
$$

where $\delta_{i j}$ is the Kronecker delta which is equal to 1 
when $i=j$ and 0 otherwise.

For the plate with a nonzero thickness, the unknowns of the potential can only be solved by using eq.(12), but for the thin plate it is not enough to obtain a unique solution, a hypersingular integral equation is required. By taking the normal derivative of the boundary integral representation of the potential function, we can obtain the hypersingular integral equation. For a boundary point $Q^{\prime}\left(\xi^{\prime}, \eta^{\prime}\right)$, it can be written as

$$
\begin{aligned}
\alpha \frac{\partial \phi_{I I}\left(\xi, \eta^{\prime}\right)}{\partial n_{f}} & =\int_{\Gamma} \phi_{I I}(\xi, \eta) \frac{\partial^{2}}{\partial n_{f} \partial n_{s}}(\ln r) d \Gamma(s) \\
& -\int_{\Gamma} \frac{\partial \phi_{I I}(\xi, \eta)}{\partial n_{s}} \frac{\partial}{\partial n_{f}}(\ln r) d \Gamma(s)
\end{aligned}
$$

where $n_{f}$ is the unit outward normal at the field point.

$\int$ denotes the Hadamard principal-value integral.

In a similar way for obtaining eq.(12), equation (15) can also be written in the matrix form as follows:

$$
\begin{aligned}
& {\left[\begin{array}{lll}
\bar{L}_{\Gamma_{n} \Gamma_{n}} & \bar{L}_{\Gamma_{n} \Gamma_{d}^{+}} & \bar{L}_{\Gamma_{n} \Gamma_{d}^{-}} \\
\dot{\bar{L}}_{\Gamma_{d}^{+} \Gamma_{n}} & \bar{L}_{\Gamma_{d}^{+} \Gamma_{d}^{+}} & \bar{L}_{\Gamma_{d}^{+} \Gamma_{d}^{-}} \\
\bar{L}_{\Gamma_{d}^{-} \Gamma_{n}} & \bar{L}_{\Gamma_{d}^{-} \Gamma_{d}^{+}} \bar{L}_{\Gamma_{d}^{-} \Gamma_{d}^{-}}
\end{array}\right]\left\{\begin{array}{l}
\left(\phi_{I I}\right)_{\Gamma_{n}} \\
\left(\phi_{I I}\right)_{\Gamma_{d}^{+}} \\
\left(\phi_{I I}\right)_{\Gamma_{d}^{-}}^{-}
\end{array}\right\}} \\
& =\left[\begin{array}{lll}
L_{\Gamma_{n} \Gamma_{n}} & L_{\Gamma_{n} \Gamma_{d}^{+}} & L_{\Gamma_{n} \Gamma_{d}^{-}} \\
L_{\Gamma_{d}^{+} \Gamma_{n}} & L_{\Gamma_{d}^{+} \Gamma_{d}^{+}} & L_{\Gamma_{d}^{+} \Gamma_{d}} \\
L_{\Gamma_{d}} \Gamma_{n} & L_{\Gamma_{d}^{-} \Gamma_{d}^{+}} & L_{\Gamma_{d}^{-} \Gamma_{d}^{-}}
\end{array}\right]\left\{\begin{array}{l}
\left(\frac{\partial \phi_{I I}}{\partial n}\right)_{\Gamma_{n}} \\
\left(\frac{\partial \phi_{I I}}{\partial n}\right)_{\Gamma_{d}^{+}} \\
\left(\frac{\partial \phi_{I I}}{\partial n}\right)_{\Gamma_{d}^{-}}
\end{array}\right\},
\end{aligned}
$$

where the elements, $L_{i j}$ and $\bar{L}_{i j}$, of the matrices of the eq.(16) are

$$
\begin{aligned}
& L_{i j}=\int_{\Gamma_{j}} \frac{\partial}{\partial n_{f}}(\ln r) d \Gamma\left(s_{j}\right)+\pi \delta_{i j}, \\
& \bar{L}_{i j}=\int_{\Gamma_{j}} \frac{\partial^{2}}{\partial n_{f} \partial n_{s}}(\ln r) d \Gamma\left(s_{j}\right) .
\end{aligned}
$$

In order to obtain a unique solution, the equations collocated on one side of the thin plate in eq.(16) must be replaced by the equations collocated on the same side of the thin plate in eq.(12), this leads to

$$
\left[\begin{array}{lll}
\bar{L}_{\Gamma_{n} \Gamma_{n}} & \bar{L}_{\Gamma_{n} \Gamma_{d}^{+}} & \bar{L}_{\Gamma_{n} \Gamma_{d}^{-}} \\
\bar{L}_{\Gamma_{d}^{+} \Gamma_{n}} & \bar{L}_{\Gamma_{d}^{+} \Gamma_{d}^{+}} & \bar{L}_{\Gamma_{d}^{+} \Gamma_{d}} \\
\bar{U}_{\Gamma_{d} \Gamma_{n}} & \bar{U}_{\Gamma_{d}^{-} \Gamma_{d}^{+}} & \bar{U}_{\Gamma_{d} \Gamma_{d}^{-}}
\end{array}\right]\left\{\begin{array}{l}
\left(\phi_{I I}\right)_{\Gamma_{n}} \\
\left(\phi_{I I}\right)_{\Gamma_{d}^{+}} \\
\left(\phi_{I I}\right)_{\Gamma_{d}^{-}}
\end{array}\right\}
$$

$$
=\left[\begin{array}{lll}
L_{\Gamma_{n} \Gamma_{n}} & L_{\Gamma_{n} \Gamma_{d}^{+}} & L_{\Gamma_{n} \Gamma_{d}^{-}} \\
L_{\Gamma_{d}^{+} \Gamma_{n}} & L_{\Gamma_{d}^{+} \Gamma_{d}^{+}} L_{\Gamma_{d}^{+} \Gamma_{d}^{-}} \\
U_{\Gamma_{d}} \Gamma_{n} & U_{\Gamma_{d}^{-}} \Gamma_{d}^{+} & U_{\Gamma_{d}^{-} \Gamma_{d}^{-}}^{-}
\end{array}\right]\left\{\begin{array}{c}
\left(\frac{\partial \phi_{I I}}{\partial n}\right)_{\Gamma_{n}} \\
\left(\frac{\partial \phi_{I I}}{\partial n}\right)_{\Gamma_{d}^{+}} \\
\left(\frac{\partial \phi_{I I}}{\partial n}\right)_{\Gamma_{d}^{-}}
\end{array}\right\} .
$$

Using boundary conditions, eqs.(2) to (8), we can obtain the following matrix form equation:

$$
\left[A^{* *}\right]\left\{\phi_{I I}\right\}=\left\{B^{* *}\right\},
$$

where $\left[A^{* *}\right]$ and $\left\{B^{* *}\right\}$ are the coefficient matrix and vector. The value of potential on the boundary of region (II) can be evaluated by solving eq.(20).

By the linearized Bernoulli equation, the nondimensional hydrodynamic pressure, $P / \rho g \zeta$, can be calculated by

$$
\frac{P}{\rho g \zeta}=-i \phi_{I I}(x, z) \exp (i \sigma t)
$$

The non-dimensional resultant uplifting force, $F / \rho g \zeta b$, acting on the thin plate can be obtained by

$$
\begin{aligned}
\frac{F}{\rho g \zeta b} & =\frac{1}{\rho g \zeta b}\left\{\int_{w}^{w+\left(b-b^{\prime}\right) / 2}\left(P_{\Gamma_{d}^{+}}-P_{\Gamma_{d}^{-}}\right) d \Gamma(s)\right. \\
& \left.+\int_{w+\left(b+b^{\prime}\right) / 2}^{w+b}\left(P_{\Gamma_{d}^{+}}-P_{\Gamma_{d}}\right) d \Gamma(s)\right\},
\end{aligned}
$$

where $P_{\Gamma_{d}^{+}}$and $P_{\Gamma_{d}}$ denote the hydrodynamic pressures on the upper and lower surfaces of the thin plate, respectively.

\section{NUMERICAL RESULTS}

The numerical results are obtained under the conditions as shown in the following table.

Table 1. Non-dimensional parameters for all examples

\begin{tabular}{|c|l|}
\hline$\sigma^{2} h / g$ & 0.25 to 2.50 with an interval 0.25 \\
\hline$d / h$ & 0.25 and 0.5 \\
\hline$b / h$ & 1.00 and 1.50 \\
\hline$w / h$ & $0.00,0.25,0.50$ and 1.00 \\
\hline$b^{\prime} / b$ & $0.0,1 / 6$ and $1 / 3$ \\
\hline
\end{tabular}

where $\sigma^{2} h / g$ is the non-dimensional frequency, $d / h$ is the non-dimensional depth of the location of the thin plate, $b / h$ is the non-dimensional length of thin plate, $w / h$ is the non-dimensional distance from edge 


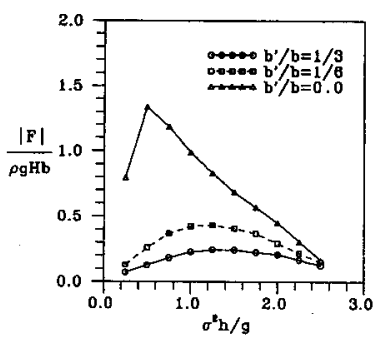

(a) $w / h=0.00$

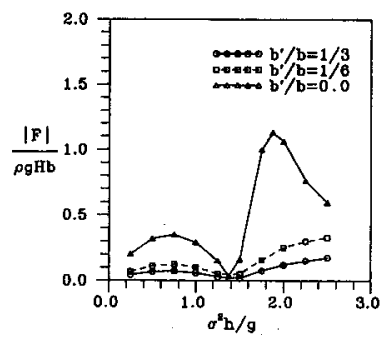

(c) $w / \mathrm{h}=0.50$

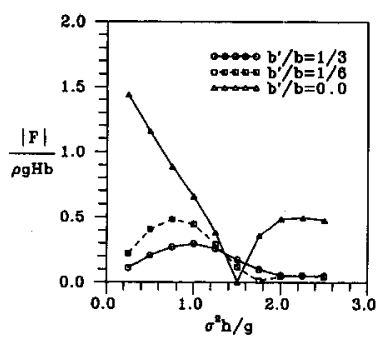

(a) $w / h=0.00$

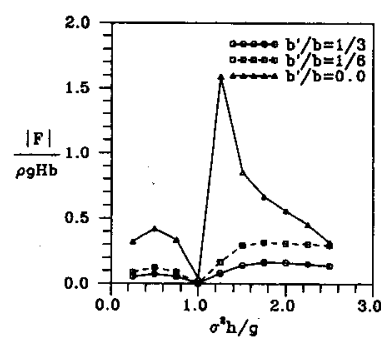

(c) $w / h=0.50$

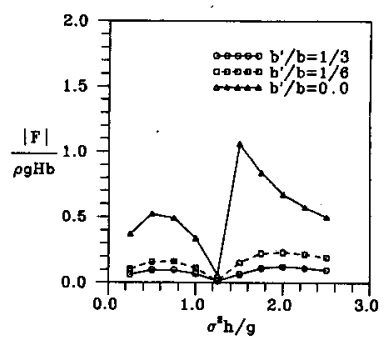

(b) $\pi / h=0.25$

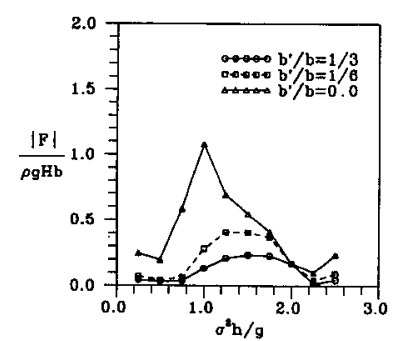

(d) $\pi / \mathrm{h}=1.00$

Fig. 2. Comparison of non-dimensional amplitudes of resultant uplifting forces. $(b / h=1.0, d / h=0.25)$

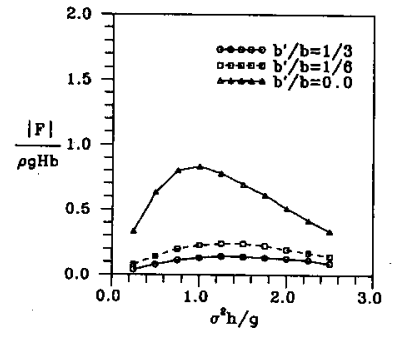

(a) $w / h=0.00$

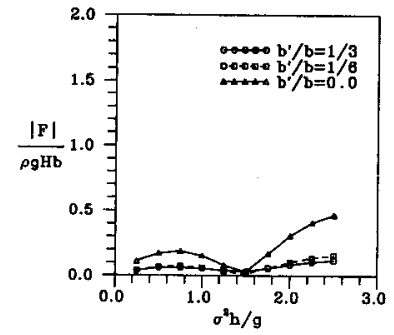

(c) $w / \mathrm{h}=0.50$ (d) $w / h=1.00$

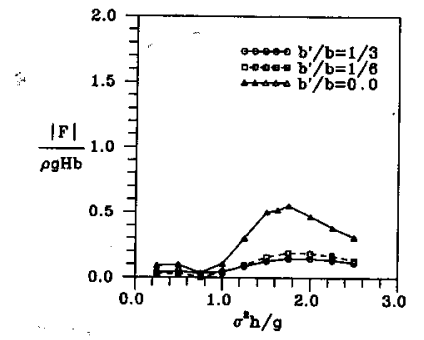

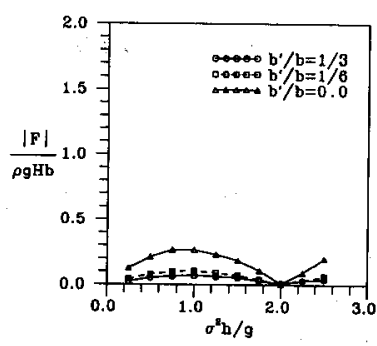

(b) $w / h=0.25$
Fig. 3. Comparison of non-dimensional amplitudes of resultant uplifting forces. $(b / h=1.0, d / h=0.50)$
Fig. 4. Comparison of non-dimensional amplitudes of resultant uplifting forces. $(b / h=1.5, d / h=0.25)$

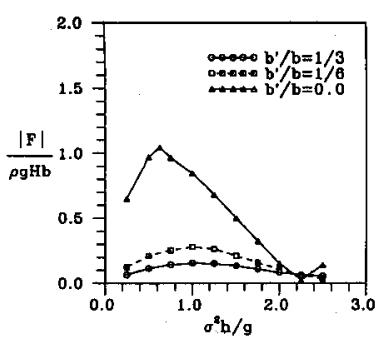

(a) $w / h=0.00$

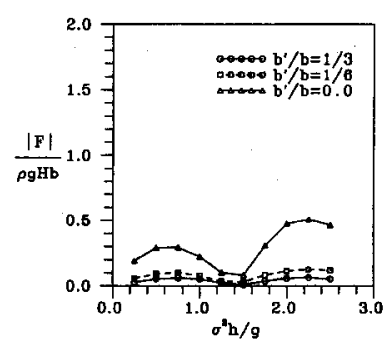

(b) $w / h=0.25$

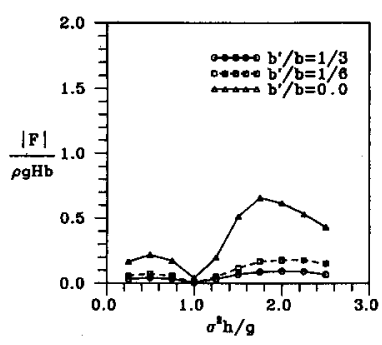

(c) $w / h=0.50$

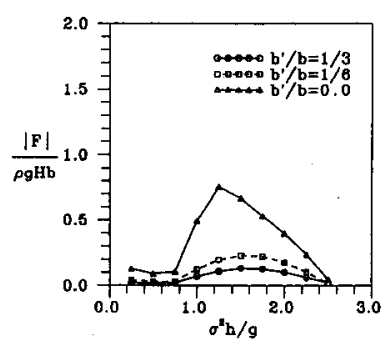

(d) $w / h=1.00$
Fig. 5. Comparison of non-dimensional amplitudes of resultant uplifting forces. $(b / h=1.5, d / h=0.50)$ 
of the thin plate to vertical wall, and $b^{\prime} / b$ is the ratio of the hole to the plate.

In different cases, the final calculated results are shown in Fig. 2(a) to 5(d). In all the figures, the non-dimensional amplitude of the resultant uplifting forces $|F| / \rho g H b$ is plotted for different values of the non-dimensional frequencies $\sigma^{2} h / g$ and of the ratio of a hole to a plate $b^{\prime} / b$.

From all the figures, it is found that the ratio of a hole to a plate $b^{\prime} / b$ decreases, the amplitude of the resultant uplifting forces on the thin plate increases. When the ratio decreases to zero, which is the case of a plate without a hole, the largest amplitude of resultant uplifting forces occurs. The effect of a hole on the uplifting force is different for different nondimensional frequencies of the water wave,

Figs. 2(a), 3(a), 4(a), and 5(a) show the cases of no distance between the edge of the thin plate and the vertical wall, $w / h=0.00$. It is found that the largest amplitude of resultant uplifting forces always occurs in the lower frequency range $\sigma^{2} h / g \leq 1.0$, otherwise, $(w / h=0.25,0.50$, and 1.00$)$, the largest amplitude of resultant uplifting forces nearly always occurs in the higher frequency range $\sigma^{2} h / g \geq 1.0$, those can be found from Figs. 2(b)-(d) to Figs.5(b)(d).

Comparing the results shown in Fig. 2 and Fig. 4 with those shown in Fig. 3 and Fig. 5 , the effect of the non-dimensional depths of the location of the thin plate, $d / h$, on the uplifting force can be observed. Therefore, the resultant uplifting forces in the cases of $d / h=0.25$ are larger than those in the cases of $d / h$ $=0.50$, and the effect of the hole on the resultant uplifting force is larger in the cases of $d / h=0.25$.

As the results shown in Fig. 2 and Fig. 3 are compared with those shown in Fig. 4 and Fig. 5, the effect of the non-dimensional lengths of thin plate, $b / h$, on the uplifting force can be seen. Thus the resultant uplifting forces of the cases of $b / h=1.5$ are larger than those of the cases of $d / h=1.0$ except the case of the comparison of Fig. 2(d) and Fig. 4(d), and the effect of the hole on the resultant uplifting force is larger in the cases of $b / h=1.5$.

\section{CONCLUSIONS}

From the above studies and discussions, some remarks are written in the following:

1. The ratio of a hole to a plate is smaller, the amplitude of resultant uplifting forces on the thin plate is larger. The largest amplitude of the resultant uplifting forces occurs in the case with no hole.

2. The effect of a hole on the uplifting forces depends on the non-dimensional frequency of the water wave.

3. When the distance between the edge of the thin plate and the vertical wall vanishes, the largest amplitude of resultant uplifting forces always occurs in the case of the plate without a hole and in the lower frequency range $\sigma^{2} h / g \leq 1.0$, otherwise, the largest amplitude of resultant uplifting forces almost always occurs in the case of the plate without a hole and in the higher frequency range $\sigma^{2} h / g \geq 1.0$.

4. When the submergence of the thin plate is deeper, the effect of a hole on the uplifting forces becomes smaller.

5. When the length of the plate is shorter, the effect of a hole on the uplifting forces becomes smaller.

\section{REFERENCES}

1. Kuo, Y.Y. \& Yueh, C.Y., "The study of wave pressure and uplift force on a submerged plate with holes," Proc. 5th Conf. on Hydraulic Engineering, pp. 910923 (1990), (in Chinese).

2. Yueh, C.Y.\& Kuo, Y.Y., "The study of wave pressure and uplift force on a submerged plate," Ocean Engineering, Vol. 20, No. 3, pp. 263-280 (1993).

3. Yueh, C.Y., "A study of wave pressure reduction acting on vertical structure using a submerged plate," Ph.D. thesis, National Chiao Tung University, Hsinchu, Taiwan, R.O.C. (1993), (in Chinese).

4. Tsaur, D.H. \& Her, S.R., "Uplift forces on a horizontal thin plate before a vertical quay," Proc. 16th Conf. on Ocean Engineering, pp. C158-C170 (1994), (in Chinese).

5. Portela, A. \& Aliabadi, M.H., "The dual boundary element method: effective implementation for crack problems," International Journal for Numerical Methods in Engineering, Vol. 33, pp. 1269-1287 (1992).

6. Chen, J.T., Hong, H.-K. \& Chyuan, S.W., "Boundary element analysis and design in seepage problems using dual integral formulation," Finite Elements in Analysis and Design, Vol. 17, pp. 1-20 (1994).

7. Subbiah, K., Shankar, N.J., \& Cheong, H.-F., "Wave forces on a large horizontal cylinder near a plane boundary," Ocean Engng., Vol. 20, No. 1, pp.77-95 (1993). 


\section{孔洞對水中水平薄板上揚力之影響}

曹登皓何世榮

國立台㩐海洋大事河海工程研究所

摘 要

本文是以透界元素法來解析及部端孔洞對水 中水平薄板上揚力之影繁。其中由於水中薄板的問 题為一退化遥界問題, 因此本文將使用超奇異皘分 方程搭配造界元素法來求解此一問題。本文中所探 用的是線性重力水波理論, 並只考虑二维的情況。 最後則以一些例子來作部算及影示孔洞對水中水平 薄板上揚力之影箱。 